\title{
Malária na região de Campinas, São Paulo, Brasil, 1980 a 1994
}

\author{
Malaria in region of Campinas, São Paulo, \\ Brazil, 1980 to 1994
Maria José Chinelatto Pinheiro Alves, Osias Rangel e Savina Silvana Aparecida Lacerra de Souza

\begin{abstract}
Resumo São apresentados dados epidemiológicos no período de 1980 a 1994 de 2.781 casos de malária assim distribuídos: DIR XII - Campinas (49,3\%), DIR XV - Piracicaba (41,3\%) e DIR $X X$ - São João da Boa Vista (9,4\%). O Plasmodium vivax foi encontrado em $70,6 \%$ dos pacientes; Plasmodium falciparum em 25,4\% e 4\% de infecção mista. Segundo a classificação epidemiológica $95 \%$ dos casos são procedentes dos Estados de Rondônia, Mato Grosso e Pará. O sexo masculino, na faixa etária de 20 a 39 anos, foi responsável por 84,3\% dos casos confirmados. No período estudado foram registrados 9 casos de malária induzida: 5 por transfusões sangüíneas, 3 pelo uso de seringas e agulhas contaminadas entre os usuários de drogas e 1 caso de malária congênita. Foram registrados 5 óbitos em doentes primo-infectados por P. falciparum com diagnóstico tardio. O conjunto das variáveis estudadas permite conhecer a epidemiologia da doença na região, subsidiar e nortear o processo de descentralização do atendimento, diagnóstico e tratamento a paciente de malária, assim como o controle e a vigilância epidemiológica da endemia na região de Campinas e no Estado de São Paulo.
\end{abstract}

Palavras-chaves: Malária. Epidemiologia. Vigilância epidemiológica.

Abstract According to epidemiological data, from 1980 to 1994, 2,781 cases of malaria were registered as follows: DIR XII - Campinas (49.3\%), DIR XV - Piracicaba (41.3\%) and DIR XX São João da Boa Vista (9.4\%). The Plasmodium vivax was found in $70.6 \%$ of the patients; Plasmodium falciparum in $25.4 \%$ and $4 \%$ of the patients presented a mixed infection. The epidemiological classification showed that $95 \%$ of the cases came from the States of RondUnia, Mato Grosso and Par. The masculine sex, between the age of 20 to 39 years old, was responsible for $84.3 \%$ of the confirmed cases. During the study period, 9 cases of induced malaria were registered: 5 of which through blood transfusion, 3 through the sharing of syringes and infected needles among drug users and one occurrence of congenital malaria. 5 deaths were registered in patients prime-infected by Plasmodium falciparum with late diagnosis. The set of variables studied enabled a greater understanding of the epidemiology of malaria in the region and to subsidize and indicate the process of decentralization of attendance, diagnosis and treatment of the patient, as well as the control and epidemiological surveillance of the endemic disease in Campinas region and in the State of São Paulo.

Key-words: Malaria. Epidemiology. Epidemiological surveillance.

Superintendência de Controle de Endemias - SUCEN - Serviço Regional 5 - Campinas, SP. Endereço para correspondência: Dr ${ }^{\mathrm{a}}$ Maria José Chinelatto P. Alves. R. São Carlos 546, Vila Industrial, 13035-420 Campinas, SP, Brasil. Telefax: 5519 272-9891/272-6823

Recebido para publicação em 13/10/98. 
A malária distribui-se pelo Continente Africano, Asiático e Americano. Continua sendo uma das mais importantes doenças infectoparasitárias, transmitida por vetor, com altos índices de morbidade e mortalidade ${ }^{813}$. Estimase registro anual de 300 a 500 milhões de casos no mundo. Destes, $90 \%$ são registrados na África tropical. Os óbitos variam entre 1,5 a 2,7 milhões de casos/ano, principalmente entre crianças menores de 2 anos $^{712}$.

Dentre os países da América, o Brasil notificou 426.510 casos de malária no ano de 1996, o maior número absoluto de casos $(39,1 \%)$, seguido por Peru (19,1\%) e Colômbia (12,3\%). Esta enfermidade representa para o Brasil um dos mais importantes problemas de Saúde Pública, vindo figurar entre as grandes endemias que assolam o país ${ }^{13}$.

A partir da $1^{\text {a }}$ metade dos anos 70 e até o final dos anos 80, houve um aumento dos casos registrados na Amazônia Legal, região que apresentou altas taxas de crescimento demográfico, influenciado, principalmente, pela construção de rodovias, aberturas de projeto de colonização do Instituto Nacional de Colonização e Reforma Agrária (INCRA) e expansão de áreas de garimpo ${ }^{10}$. No início dos anos 90 , observa-se na região amazônica a estabilização e até mesmo um ligeiro declínio no número de casos confirmados. A situação se mantém oscilante até 1996. Algumas explicações possíveis para esta situação seriam a redução de deslocamentos para área endêmica em virtude de dificuldades econômicas; esgotamento de áreas de extração mineral; estabilidade da população em projetos agrícolas e utilização de esquemas terapêuticos mais eficientes ${ }^{10}{ }^{19}$. Salienta-se que a região amazônica concentra mais de $99 \%$ da transmissão natural da malária no Brasil.

No Estado de São Paulo, até 1987, a tendência do número de casos foi semelhante ao observado no país. A partir de 1988 e até 1994, há um registro de queda acentuada anual. Esta diminuição poderia estar relacionada com a estabilização da área endêmica - região amazônica — de onde procede a maioria dos doentes de malária diagnosticados no Estado. São representados por indivíduos que se deslocaram para aquela região à procura de novas oportunidades de vida e emprego, principalmente nas grandes áreas de extração mineral ${ }^{118}$.

A região de Campinas reflete a mesma situação observada para o Estado de São Paulo. Os casos de malária, em sua maioria, são importados da região amazônica e referem-se aos indivíduos aqui residentes e que para lá se deslocaram em busca de melhores condições de vida e de trabalho e, não alcançando seus objetivos, retornaram para suas residências ${ }^{123}$. As áreas com transmissão local, como o complexo da Mata Atlântica e região do Planalto Paulista, são pouco significativas ${ }^{519} 23$.

Este trabalho tem como objetivo conhecer a epidemiologia da malária, promover o controle e vigilância epidemiológica da endemia, subsidiar e nortear o processo de descentralização do atendimento, diagnóstico e tratamento do paciente na região de Campinas que, na divisão administrativa do Estado em 10 regiões, corresponde a 5a região, denominada Serviço Regional 5 - SR 5 - Campinas, da Superintendência de Controle de Endemias - SUCEN.

\section{MATERIAL E MÉTODOS}

O presente estudo foi realizado na região de Campinas, composta por 88 municípios, distribuídos em 3 Divisões Regionais de Saúde (DIR's): Campinas (DIR XII) - 38 municípios, Piracicaba (DIR XV) - 25 municípios e São João da Boa Vista (DIR XX) - 25 municípios (Figura 1). A população desta região corresponde a aproximadamente 5.000.000 habitantes.

Nos municípios de Campinas, Mogi Guaçú e São João da Boa Vista estão instalados os laboratórios do Serviço Regional, que realizam o diagnóstico de malária pelo método de Walker ${ }^{18}$. As lâminas são provenientes de indivíduos que procuram espontaneamente os laboratórios ou são notificados através dos serviços de saúde. Após a confirmação do caso, inicia-se a investigação e classificação epidemiológica, segundo procedimentos descritos em trabalhos anteriores ${ }^{5223}$.

Foram estudados 2.781 casos de malária no período de 1980 a 1994, através da análise das fichas de investigação epidemiológica, onde se procurou avaliar as seguintes informações: sexo, faixa etária, espécie de plasmódio, local de infecção, modo de transmissão, intervalo decorrido entre os primeiros sintomas e o diagnóstico, atividade econômica no momento da transmissão, motivo do retorno para o Estado de São Paulo e evolução da doença. 


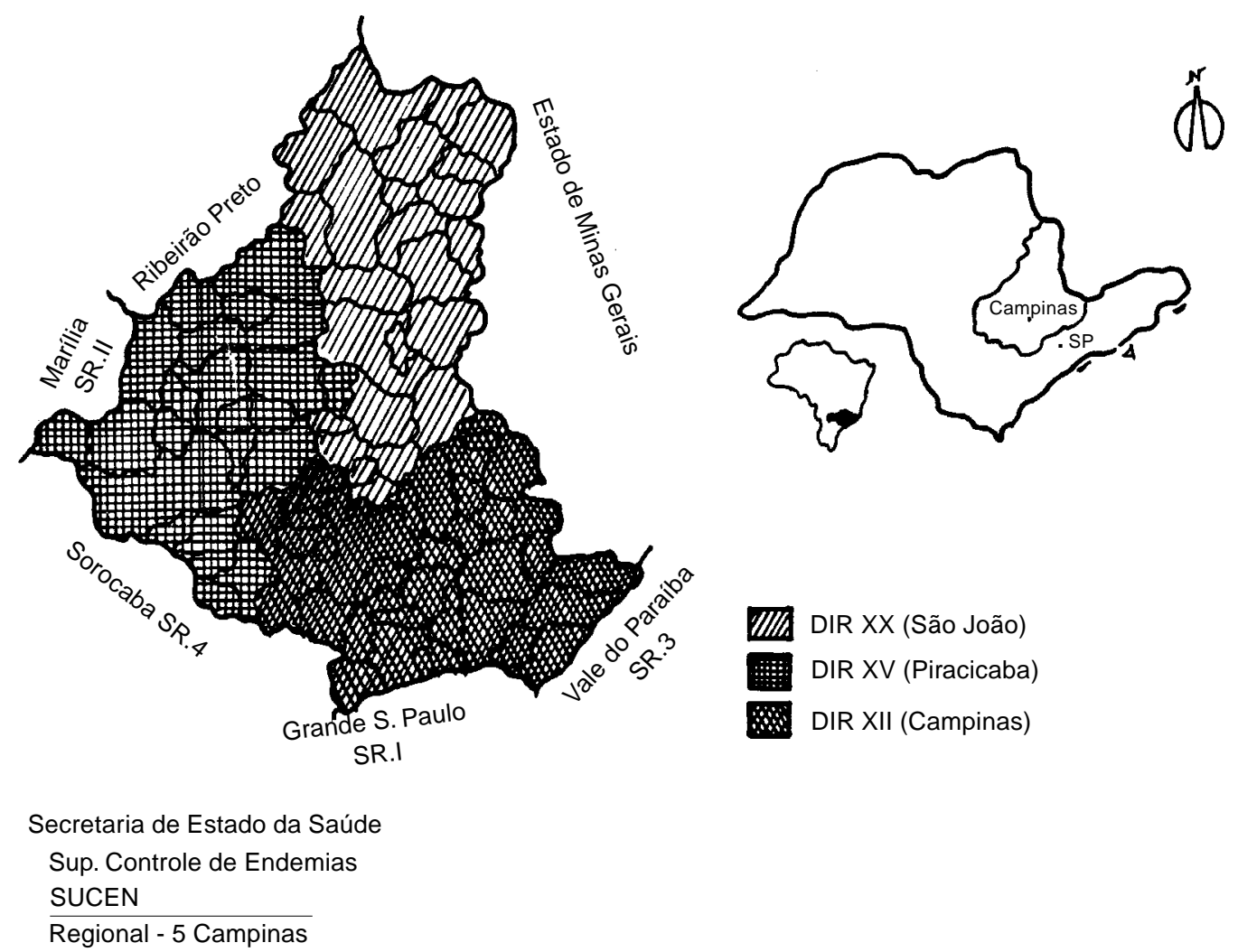

Figura 1 - Área do Estado de São Paulo correspondente ao Serviço Regional de Campinas, SP - SUCEN.

\section{RESULTADOS}

Dos 2.781 casos investigados, $70,6 \%$ a infecção foi por Plasmodium vivax, 25,4\% por Plasmodium falciparum e $4 \%$ de malária mista (Tabela 1).

Quando se analisa a distribuição dos casos segundo o intervalo entre os primeiros sintomas e diagnóstico, intervalo este já apresentado em trabalho anterior ${ }^{21}$, observa-se que não existe diferença significativa no decorrer do período estudado. A detecção precoce da doença ocorreu em $61,6 \%$ dos doentes atendidos. Esta refere-se à procura de atendimento para diagnóstico nos 3 primeiros dias de sintomas, indicando o conhecimento da sintomatologia malárica e do atendimento pela SUCEN para o diagnóstico e tratamento. A procura intermediária - 4 a 9 dias - esteve presente em $30,2 \%$ dos atendimentos realizados e a procura tardia - mais de 10 dias - em 9,2\%. Nestas duas últimas categorias acentua-se a possibilidade de gravidade em relação ao prognóstico, para os casos de malária induzida entre usuários de drogas injetáveis ${ }^{4} 9$ e nos casos de malária por P. falciparum.

A malária incidiu sobre ambos os sexos, com predomínio do masculino $(85,6 \%)$ e em todas as faixas etárias, com maior freqüência entre os indivíduos entre 20 a 39 anos (61,9\%) (Figura 2). Com referência ao local de residência, observase que $49,3 \%$ dos indivíduos moravam em municípios pertencentes à DIR XII, 41,3\% à DIR XV e $9,4 \%$ à DIR XX.

Os casos importados representaram $95 \%$ do total dos casos confirmados. A procedência destes doentes (Figura 3) indica os Estados de Rondônia, Mato Grosso e Pará como os principais envolvidos na transmissão. As ocupações referidas com maior freqüência nos locais de transmissão foram a extração mineral $(25,3 \%)$, agropecuária $(20,4 \%)$ e transporte $(10,8 \%)$. 
Tabela 1 - Casos investigados de malária, segundo espécie de plasmódio. Região de Campinas, 1980 a 1994.

\begin{tabular}{|c|c|c|c|c|c|c|c|}
\hline \multirow[t]{2}{*}{ Ano } & \multicolumn{2}{|c|}{ P. vivax } & \multicolumn{2}{|c|}{ P. falciparum } & \multicolumn{2}{|c|}{$P . v+P . f$} & \multirow[t]{2}{*}{ Total } \\
\hline & $\mathrm{n}^{0}$ & $\%$ & $\mathrm{n}^{\circ}$ & $\%$ & $\mathrm{n}^{\circ}$ & $\%$ & \\
\hline 1980 & 100 & 86,2 & 16 & 13,8 & - & - & 116 \\
\hline 1981 & 96 & 70,6 & 34 & 25,0 & 6 & 4,4 & 136 \\
\hline 1982 & 93 & 74,4 & 31 & 24,8 & 1 & 0,8 & 125 \\
\hline 1983 & 131 & 67,1 & 58 & 29,8 & 6 & 3,0 & 195 \\
\hline 1984 & 147 & 69,3 & 58 & 27,3 & 7 & 3,3 & 212 \\
\hline 1985 & 134 & 60,7 & 81 & 36,7 & 6 & 2,8 & 221 \\
\hline 1986 & 190 & 66,7 & 68 & 23,9 & 27 & 9,4 & 285 \\
\hline 1987 & 211 & 68,6 & 75 & 24,3 & 22 & 7,1 & 308 \\
\hline 1988 & 161 & 68,6 & 68 & 29,0 & 6 & 2,6 & 235 \\
\hline 1989 & 179 & 70,0 & 57 & 22,2 & 20 & 7,9 & 256 \\
\hline 1990 & 150 & 74,7 & 47 & 23,3 & 4 & 2,0 & 201 \\
\hline 1991 & 114 & 74,0 & 35 & 22,8 & 5 & 3,2 & 154 \\
\hline 1992 & 111 & 78,8 & 30 & 21,2 & - & - & 141 \\
\hline 1993 & 61 & 73,5 & 21 & 25,3 & 1 & 1,2 & 83 \\
\hline 1994 & 84 & 74,3 & 29 & 25,7 & - & - & 113 \\
\hline Total & 1.962 & 70,6 & 708 & 25,4 & 111 & 4,0 & 2.781 \\
\hline
\end{tabular}

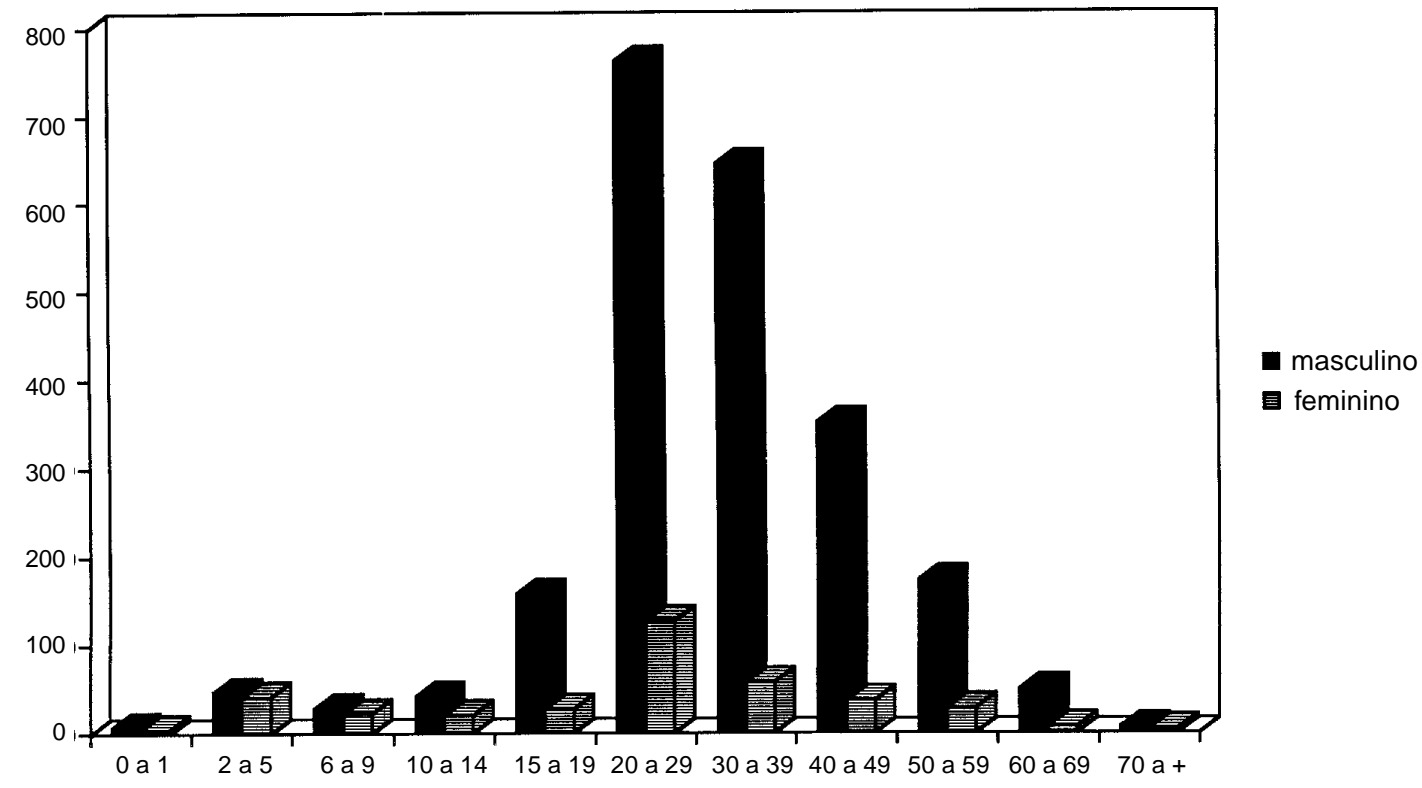

Figura 2 - Distribuição percentual dos casos de malária, segundo sexo e faixa etária. Região de Campinas, SP, 1980 a 1994.

Salienta-se que 14,8\% dos indivíduos declararam que o motivo do deslocamento para área de transmissão foi o lazer.

Os casos autóctones representaram $0,18 \%$ do total dos casos confirmados (Tabela 2). O P. vivax foi diagnosticado em 4 pacientes e o $P$. falciparum em 1 paciente. Dos 5 casos registrados, 3 referemse a pacientes com diagnóstico realizado neste Serviço Regional, porém a transmissão ocorreu em municípios do Vale do Paraíba e Vale do 


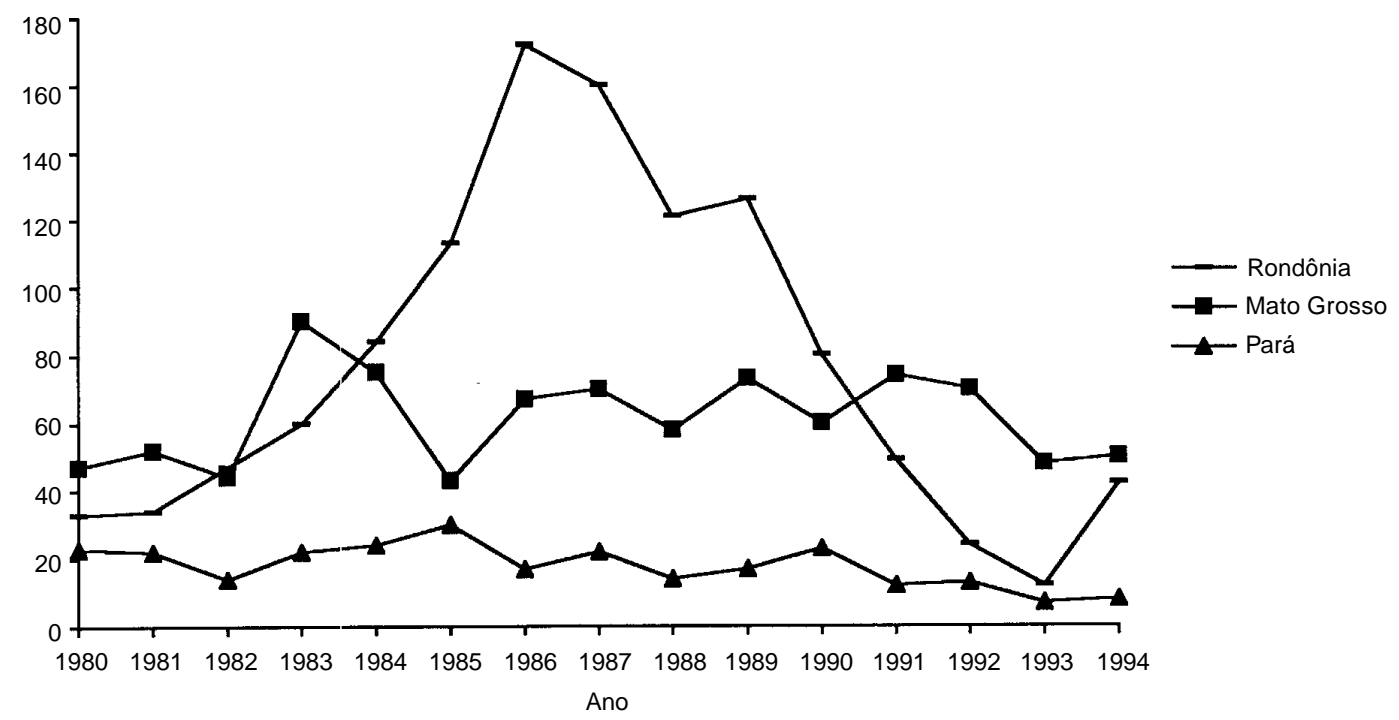

Figura 3 - Procedência dos casos importados de malária. Região de Campinas, SP, 1980 a 1994.

Tabela 2 - Distribuição dos casos de malária segundo a classificação epidemiológica. Região de Campinas, SP, 1980 a 1994

\begin{tabular}{|c|c|c|c|c|c|c|c|c|c|c|}
\hline \multirow[t]{2}{*}{ Ano } & \multirow{2}{*}{$\begin{array}{l}\text { № de } \\
\text { casos }\end{array}$} & \multirow{2}{*}{$\begin{array}{c}\% \text { de } \\
\text { variação }\end{array}$} & \multicolumn{2}{|c|}{ Importado } & \multicolumn{2}{|c|}{ Autóctone } & \multicolumn{2}{|c|}{ Induzido } & \multicolumn{2}{|c|}{ Recaída } \\
\hline & & & $\mathrm{n}^{0}$ & $\%$ & $\mathrm{n}^{\circ}$ & $\%$ & $\mathrm{n}^{\circ}$ & $\%$ & $n^{0}$ & $\%$ \\
\hline 1980 & 116 & - & 116 & 100,0 & - & - & - & - & - & - \\
\hline 1981 & 136 & 17,20 & 134 & 98,6 & 1 & 0,7 & 1 & 0,7 & - & - \\
\hline 1982 & 125 & $-8,00$ & 125 & 100,0 & - & - & - & - & - & - \\
\hline 1983 & 195 & 56,00 & 193 & 99,0 & 1 & 0,5 & 1 & 0,5 & - & - \\
\hline 1984 & 212 & 8,70 & 204 & 96,2 & - & - & 1 & 0,4 & 7 & 3,3 \\
\hline 1985 & 221 & 4,24 & 213 & 96,3 & - & - & - & - & 8 & 3,7 \\
\hline 1986 & 285 & 28,90 & 271 & 95,0 & - & - & - & - & 14 & 5,0 \\
\hline 1987 & 308 & 8,07 & 291 & 94,4 & 1 & 0,3 & - & - & 16 & 5,1 \\
\hline 1988 & 235 & $-23,70$ & 219 & 93,2 & - & - & - & - & 16 & 6,8 \\
\hline 1989 & 256 & 8,93 & 242 & 94,6 & 0 & 0 & 1 & 0,4 & 13 & 5,0 \\
\hline 1990 & 201 & $-21,40$ & 190 & 94,6 & - & - & 1 & 0,5 & 10 & 5,0 \\
\hline 1991 & 154 & $-23,30$ & 142 & 92,2 & 1 & 0,7 & 2 & 1,4 & 9 & 5,9 \\
\hline 1992 & 141 & $-8,44$ & 119 & 84,4 & - & - & - & - & 22 & 15,7 \\
\hline 1993 & 83 & $-41,13$ & 76 & 91,6 & 1 & 1,2 & 2 & 2,4 & 4 & 4,8 \\
\hline 1994 & 113 & 36,14 & 109 & 96,4 & - & - & - & - & 4 & 3,6 \\
\hline Total & 2.781 & - & 2.644 & 95,0 & 5 & 0,1 & 9 & 0,3 & 123 & 4,4 \\
\hline
\end{tabular}

Ribeira, regiões reconhecidamente de importância epidemiológica, e com a presença do Anopheles (K.) cruzii e (K.) belattor ${ }^{18}$. O 4ํㅜㄹ caso autóctone, diagnosticado em 1983, com transmissão no município de Campinas, é o primeiro registro de autoctonia nas últimas três décadas. Na elaboração do Plano de Erradicação da Malária do Estado de São Paulo ${ }^{15}$, este município não foi incluído como sendo área malárica porque a transmissão já não existia. No entanto, a pesquisa entomológica realizada em 1983, indicou razoável densidade anofélica, com 
predomínio de Anopheles (N.) albitarsis, vetor secundário, presente em todos os estados e, possivelmente o responsável pela transmissão daquele caso ${ }^{16}{ }^{18}$. O 5을 caso autóctone, infecção por $P$. falciparum, transmissão no município de Mogi Guaçú, em 1987, recebeu esta classificação epidemiológica em virtude de terem sido encontrados exemplares de Anopheles (N.) albitarsis ${ }^{17} 18$ nas margens do riacho, próximo à residência do doente.

Foram diagnosticados 9 casos de malária induzida (Tabela 2). Destes, 5 pacientes contraíram a doença por transfusão sanguínea, infecção por $P$. vivax. Três eram do sexo masculino com idade superior a 65 anos e 2 mulheres tinham idade acima de 32 anos. O intervalo decorrido entre os primeiros sintomas e o diagnóstico foi em torno de 13 dias. A malária transmitida pelo uso compartilhado de seringas e agulhas contaminadas entre usuários de droga injetável, foi detectada em 3 pacientes jovens: 2 desempregados e uma prostituta. Dois deles eram portadores de malária por $P$. vivax e um por $P$. falciparum. Este, era um garoto de 17 anos, desempregado, residente em Aguaí, SP, município próximo a Campinas. Teve atendimento médico hospitalar em 3 diferentes unidades de saúde, inclusive com encaminhamento para internação com suspeita diagnóstica de hepatite. A SUCEN foi acionada e o exame de gota espessa indicou malária por $P$. falciparum. A investigação epidemiológica permitiu concluir que o paciente era usuário de droga injetável em grupo, e neste, encontrava-se uma pessoa que morava em Mato Grosso, MT e fazia freqüentes viagens à Aguaí, SP e era portador de malária falcípara, diagnosticada por esse Serviço Regional. O caso de transmissão congênita foi assim considerado em razão da precocidade dos sintomas, ausência de transmissão vetorial no município de permanência e o recém-nascido, de 14 dias, não ter sido submetido a punções venosas ou transfusões sanguíneas ${ }^{2}$.

Os casos classificados como recaída foram em torno de $6 \%$, exceção feita a 1992, que representou $15,8 \%$ dos casos classificados. Foram descartadas as possibilidades relacionadas à qualidade e validade dos medicamentos utilizados, bem como a afirmativa do paciente de que tomou a medicação completa. Este aumento percentual pode ser explicado pelo registro de 4 casos de malária com 2 recaídas cada caso e 1 deles com 3 recaídas. Estas ocorrências não tiveram registros em anos anteriores.

Foram registrados 5 casos, infecção por $P$. falciparum que evoluíram para óbito. A investigação epidemiológica indicou que os casos eram importados de Mato Grosso, MT, do sexo masculino. Os indivíduos se deslocaram para a área de transmissão em atividade de lazer, 2 casos e para a extração mineral 3 casos. 0 intervalo entre os sintomas e o diagnóstico foi em média de 10 dias. O coeficiente de letalidade correspondeu a 1,8/1000 casos. Calculado apenas para os pacientes portadores de malária por $P$. falciparum, o coeficiente foi de $39,7 / 1000$ casos. Estes dados diferem do achado para a região de Ribeirão Preto $(21,1 / 1000 \text { casos })^{5}$ e para 0 Estado de São Paulo (14,6/1000 casos) ${ }^{23}$.

\section{DISCUSSÃO}

Os estudos de casos de malária registrados no Brasil, apontam que em torno de $99 \%$ deles foram detectados na Amazônia Legal. Esta situação repercute no Estado de São Paulo onde, $93,5 \%$ dos casos diagnosticados procederam daquela região1 192223 .

Quando se observa a distribuição dos doentes de malária na região de Campinas, notase que até 1989 houve pequenas oscilações anuais, com tendência de crescimento do número de casos. A partir de 1990 registra-se a redução (Tabela 2), como no Estado de São Paulo e região de Ribeirão Preto ${ }^{18} 22$ 23. No entanto, a situação preocupante em que se encontra a transmissão nas Américas ${ }^{13}$ e no Brasil ${ }^{10}$, coloca o Estado de São Paulo e a região de Campinas em contínuo sistema de vigilância sobre as fontes de infecção importada. Esta vigilância é fundamental nos municípios pertencentes à DIR XII e DIR XV, pois são regiões consideradas pólos de atração econômica.

O estudo realizado para delinear o perfil do portador desta doença, aponta maior percentual de indivíduos do sexo masculino, na idade economicamente produtiva, resultante do deslocamento de grupos populacionais em busca de emprego ou para desenvolver o seu trabalho nas áreas com risco de transmissão. Resultados semelhantes foram encontrados em trabalhos já publicados $^{152223}$. O conhecimento dessas variáveis é fundamental para o trabalho de divulgação junto às populações de risco e da categoria médica, uma vez que o diagnóstico de malária requer um alto índice de suspeita e a 
habilidade de reconhecer o parasito na amostra do sangue periférico ${ }^{2}$.

Se por um lado existe um grande contingente de pessoas que já conhecem e procuram o Serviço da SUCEN logo após os primeiros sinais da infecção ${ }^{21} 23$, os indivíduos, primo-infectados, por desconhecimento da doença e da prestação deste Serviço, podem permanecer sem diagnóstico por um período mais longo ${ }^{21}$ e agravar o quadro inicial, podendo, inclusive, levar a óbito, se for portador de infecção por $P$. falciparum.

A dificuldade em estabelecer a suspeita clínica precoce para malária por alguns profissionais da área médica, pode se agravar em razão da pouca relevância do estudo desta endemia nas escolas de medicina; da redução de indivíduos doentes que chegam nesta região à procura de atendimento e diagnóstico; da urbanização da malária por usuários de drogas injetáveis, através do uso de seringas e agulhas contaminadas $^{43}$ e redução das atividades educativas para a população de risco por parte do órgão responsável pelo controle da malária no Estado de São Paulo. Este aspecto é conseqüência das atividades desenvolvidas para o controle dos vetores de febre amarela e dengue, que esgota quase totalmente a capacidade operacional da SUCEN.

A malária no Estado de São Paulo apresenta uma situação epidemiológica particular, ou seja, áreas onde a transmissão foi interrompida nas décadas de 60-70, conservando porém o potencial malarígeno, podendo originar a transmissão eventual em áreas restritas. Atualmente a SUCEN não detém informações suficientes da distribuição do vetor nesta região e no Estado. Assim, a descentralização do atendimento da endemia poderia estar vinculada à realização do mapeamento das espécies vetoras de anofelinos com potencial de transmissão da doença em todo Estado de São Paulo. Este mapeamento poderia, inclusive, ajudar a entender a transmissão autóctone, por $P$. falciparum, em 1987, registrada em uma área onde, na pesquisa entomológica realizada por ocasião do foco, só foi encontrado o vetor secundário da malária.

O Estado de São Paulo deve estabelecer ações diferenciadas de controle ${ }^{23}$, direcionando os pacientes para as principais portas de entrada dos serviços de pronto socorro e pronto atendimento de hospitais e ambulatórios além de promover a inter-relação com as atividades de controle executadas por esta Instituição. Essas ações deverão resultar na descentralização do atendimento ao paciente de malária, do diagnóstico laboratorial, do tratamento específico e da investigação epidemiológica ${ }^{6}$, resultando, inclusive, no direcionamento para a prevenção de epidemias em regiões com potencial de transmissão ou manter interrompida a transmissão.

De acordo com a orientação da Organização Mundial de Saúde ${ }^{11}$, a horizontalização das ações para o controle da malária é ainda um objeto a ser atingido. Este programa, no Brasil, está seguindo as recomendações da Reunião Ministerial de Amsterdã em 1992, a qual prioriza a prevenção da mortalidade e redução da morbidade e das perdas sociais e econômicas produzidas pela doença, por meio do fortalecimento progressivo dos níveis regionais e locais ${ }^{10}$. Os objetivos a serem alcançados seriam a promoção do diagnóstico precoce e tratamento imediato; vigilância epidemiológica para prevenção ou detecção de epidemias; monitoramento dos fatores ecológicos, sociais e econômicos que determinam a doença e medidas preventivas relacionadas ao vetor.

\section{AGRADECIMENTOS}

Ao Pesquisador Científico Araripe Pacheco Dutra pelas sugestões ao trabalho, à Sandra Regina da Silva pela digitação e aos auxiliares de laboratório do SR 5 pela apropriação dos dados.

\section{REFERÊNCIAS BIBLIOGRÁFICAS}

1. Alves MJCP, Barata LCB, Barata RCB, Almeida MCRR, Gutierrez EB, Wanderley DMV, Andrade JCR. Aspectos sócio-econômicos dos indivíduos com malária importada na região metropolitana de São Paulo, Brasil. I Caracterização da população e conhecimento sobre a doença. Revista de Saúde Pública de São Paulo 24:253258, 1990.
2. Alves MJCP, Lima VLC, Rangel O. Malária congênita no município de Leme, SP. Jornal de Pediatria 71:163-165, 1995.

3. Andrade JCR, Wanderley DMV. Malária induzida no Estado de São Paulo, Brasil. Revista da Sociedade Brasileira de Medicina Tropical 24:157-161, 1991. 
4. Barata LCB, Andriguetti MTM, Matos MR. Surto de malária induzida entre usuários de drogas injetáveis. Revista de Saúde Pública 27:9-14, 1993.

5. Capuano DM, Gomes AS, Polidorio SRB. Malária na região de Ribeirão Preto - São Paulo, Brasil - 1984 a 1993. Revista do Instituto Adolfo Lutz 56:1-8, 1996.

6. Diário Oficial do Estado de São Paulo. Secretaria de Estado da Saúde. Resolução SS-5, de 8/1/98 9/1/98, Seção 1, n 6, p.14, 1998.

7. Fundação Nacional de Saúde. Informe epidemiológico do SUS- Ministério da Saúde (Brasil), ano V noำ out. a dez, 1996.

8. Fundação Nacional de Saúde. Centro Nacional de Epidemiologia. Boletim Epidemiológico. Ministério da Saúde (Brasil), ano III, ㄲo 6, 1997.

9. Loo SS, Andrade JCR, Condino MLF, Alves MJCP, Semeghini MG, Galvão EC. Malária em usuários de drogas de administração endovenosa associada à soropositividade para HIV. Revista de Saúde Pública, São Paulo 25:17-22, 1991.

10. Marques AC, Gutierrez HC. Combate à malária: evolução, situação atual e perspectivas. Revista da Sociedade Brasileira de Medicina Tropical 27 (supl III): 91-108, 1994 .

11. Najera JA. Malaria and the work of World Health Organization. Bulletin 67:229-243, 1989.

12. Organización Mundial de la Salud. DTHE World Health Reports, 1998

13. Organizacion Panamericana de la Salud. Situación de la malaria em las Américas, vol. 18, $\mathrm{n} \cong 3,1996$.

14. Sawyer DR. Aspectos sociais da malária no Brasil. Belo Horizonte, CEDEPLAR/UFMG, (relatório de pesquisa), 1987.
15. Serviço de Profilaxia da Malária do Governo do Estado de São Paulo. Plano para a Erradicação da Malária no Estado de São Paulo - Brasil (mímeo), 1958.

16. Superintendência de Controle de Endemias. Relatório de avaliação epidemiológica do Programa de Erradicação da Malária no Estado de São Paulo. Secretaria de Estado da Saúde de São Paulo (mimeo), São Paulo, 1983.

17. Superintendência de Controle de Endemias. Relatório de avaliação epidemiológica do Programa de Erradicação da Malária no Estado de São Paulo, Secretaria de Estado da Saúde de São Paulo (mimeo), São Paulo, 1987.

18. Superintendência de Controle de Endemias. Malária Situação atual, diagnóstico, tratamento e controle. Secretaria de Estado da Saúde de São Paulo, 1988.

19. Superintendência de Controle de Endemias. Avaliação do Programa de Controle de Malária - Estado de São Paulo. Secretaria de Estado da Saúde de São Paulo, 1992 a 1994.

20. Tauil PL. Malária: agrava-se o quadro da doença no Brasil. Ciência Hoje 2:58-64, 1984.

21. Wanderley DMV, Andrade JCR, Alves MJCP, Alves MCGP, Mattos MR, Gurgel SM, Igreja RP. Malária no Estado de São Paulo: Avaliação de aspectos da vigilância epidemiológica. Caderno de Saúde Pública 5:296-304, 1989.

22. Wanderley DMV, Andrade JCR, Meneguetti LC, Chinelatto MJ, Dutra AP. Malária no Estado de São Paulo, Brasil, 1980 a 1983. Revista de Saúde Pública 19:2836, 1985.

23. Wanderley DMV, Silva RA, Andrade JCR. Aspectos epidemiológicos da malária no Estado de São Paulo, Brasil - 1983 a 1992. Revista de Saúde Pública 28:192197, 1994 . 